\title{
ON COMPACT RIEMANNIAN MANIFOLDS WITH HARMONIC WEYL CURVATURE
}

\author{
HAI-PING FU AND HUI-YA HE*
}

\begin{abstract}
Aвstract. We give some rigidity theorems for an $\mathrm{n}(\geq 4)$-dimensional compact Riemannian manifold with harmonic Weyl curvature, positive scalar curvature and positive constant $\sigma_{2}$. Moreover, when $n=4$, we prove that a 4-dimensional compact locally conformally flat Riemannian manifold with positive scalar curvature and positive constant $\sigma_{2}$ is isometric to a quotient of the round $\mathbb{S}^{4}$.
\end{abstract}

\section{INTRODUCTION AND MAIN RESULTS}

Recall that an $n$-dimensional Riemannian manifold $\left(M^{n}, g\right)(n \geq 4)$ is said to be a manifold with harmonic Weyl curvature (resp. harmonic curvature) if the divergence of its Weyl curvature tensor $W$ vanishes, i.e., $\delta W=0$ (resp. the divergence of its Riemannian curvature tensor $R m$ vanishes, i.e., $\delta R m=0$ ). In view of the second Bianchi identity, we know that $M^{n}$ has harmonic Weyl curvature (resp. harmonic curvature) if and only if the Schouten tensor (resp. the Ricci tensor) of $M^{n}$ is a Codazzi tensor. By the Bianchi identity, the scalar curvature on manifolds with harmonic curvature is constant. $M^{n}$ has harmonic curvature if and only if $M^{n}$ has harmonic Weyl curvature and constant scalar curvature. Thus, every Riemannian manifold with parallel Ricci tensor has harmonic curvature. The locally conformally flat Riemannian manifolds and the products of Einstein manifolds are also important examples of manifolds with harmonic Weyl curvature, however, the converse does not hold (see [2], for example). According to the decomposition of the Riemannian curvature tensor, the metric with harmonic Weyl curvature is a natural candidate for this study since one of the important problems in Riemannian geometry is to understand classes of metrics that are, in some sense, close to being Einstein or having constant curvature.

The complete manifolds with harmonic curvature have been studied in literature (e.g., [5, 6, 9, 10, 11, 12, 13, 14, 17, 20, 22, 24, 27, 29, 30, 32]). Some isolation theorems of Weyl curvature tensor of positive Einstein manifolds are given in [7, 14, 17, 20, 29], when its $L^{p}$-norm is small. Some scholars classify conformally flat manifolds satisfying some curvature $L^{p}$-pinching conditions [6, 12, 13, 17, 27, 32]. Recently, Tran [31] obtain two rigidity results for a closed Riemannian manifold with harmonic Weyl curvature, which are a generalization of Tachibana's theorem for non-negative curvature operator [30] and integral gap result which extends Theorem 1.10 for manifolds with harmonic curvature in [11]. We are interested in some pinching problems for compact Riemannian manifold with harmonic Weyl curvature.

2000 Mathematics Subject Classification. Primary 53C21; Secondary 53C20.

Key words and phrases. Einstein manifold, harmonic Weyl curvature, Schouten tensor.

Supported by National Natural Science Foundations of China \#11761049, Jiangxi Province Natural Science Foundation of China \#20171BAB201001. 
Now we introduce the definition of the Yamabe constant. Given a compact Riemannian manifold $\left(M^{n}, g\right)(n \geq 3)$, we consider the Yamabe functional

$$
Q_{g}: C_{+}^{\infty}\left(M^{n}\right) \rightarrow \mathbb{R}: f \mapsto Q_{g}(f)=\frac{\frac{4(n-1)}{n-2} \int_{M^{n}}|\nabla f|^{2} \mathrm{~d} v_{g}+\int_{M^{n}} R f^{2} \mathrm{~d} v_{g}}{\left(\int_{M^{n}} f^{\frac{2 n}{n-2}} \mathrm{~d} v_{g}\right)^{\frac{n-2}{n}}},
$$

where $R$ denotes the scalar curvature of $M^{n}$. It follows that $Q_{g}$ is bounded below by Hölder inequality. We set

$$
Y\left(M^{n},[g]\right)=\inf \left\{Q_{g}(f) \mid f \in C_{+}^{\infty}\left(M^{n}\right)\right\} .
$$

This constant $Y\left(M^{n},[g]\right)$ is an invariant of the conformal class of $\left(M^{n}, g\right)$, called the Yamabe constant. The important works of Aubin, Schoen, Trudinger and Yamabe showed that the infimum in $\inf \left\{Q_{g}(f) \mid f \in C_{+}^{\infty}\left(M^{n}\right)\right\}$ is always achieved (see [1, 23]). The Yamabe constant of a given compact manifold is determined by the sign of scalar curvature [1]. For a Riemannian manifold $\left(M^{n}, g\right)(n \geq 3)$, we denote by $\sigma_{2}\left(A_{g}\right)$ the second elementary symmetric function of the eigenvalues of the so-called Schouten tensor $A_{g}=R i c-\frac{R}{2(n-1)} g$ with respect to $g$. Hence

$$
2 \sigma_{2}\left(A_{g}\right)=\left(\operatorname{tr} A_{g}\right)^{2}-\left|A_{g}\right|^{2}=\frac{(n-2)^{2}}{4 n(n-1)} R^{2}-\left|R_{i c}\right|^{2},
$$

where Ric denotes the trace-free Ricci tensor.

In this note, we obtain the following rigidity theorems.

Theorem 1.1. Let $\left(M^{n}, g\right)(n \geq 4)$ be an $n$-dimensional compact Riemannian manifold with harmonic Weyl curvature, positive scalar curvature and positive constant $\sigma_{2}\left(A_{g}\right)$. If

$$
|W|^{2}+\frac{2 n^{2}(n-4)}{(n-2)^{3}}|R i c|^{2}<\frac{16 n}{(n-2)^{3}} \sigma_{2}\left(A_{g}\right),
$$

then $\left(M^{n}, g\right)$ is an Einstein manifold.

Remark 1.2. The inequality (1.2) of this theorem is optimal. The critical case is given by the product manifold $\mathbb{S}^{1} \times \mathbb{S}^{n-1}$.

Theorem 1.3. Let $\left(M^{n}, g\right)(n \geq 4)$ be an $n$-dimensional compact Riemannian manifold with harmonic Weyl curvature, positive scalar curvature and positive constant $\sigma_{2}\left(A_{g}\right)$. If

$$
|W|^{2}+\left[\frac{2 n}{n-2}-\frac{16(n-1)}{n(n-2)^{2} C(n)^{2}}\right]|R i c|^{2}<\frac{32(n-1)}{n(n-2)^{2} C(n)^{2}} \sigma_{2}\left(A_{g}\right)
$$

where

$$
C(n)=\left\{\begin{array}{l}
\frac{\sqrt{6}}{2}, \quad \text { for } n=4, \\
\frac{8}{\sqrt{10}}, \quad \text { for } n=5, \\
\frac{2(n-2)}{\sqrt{n(n-1)}}+\frac{n^{2}-n-4}{\sqrt{(n-2)(n-1) n(n+1)}}, \quad \text { for } n \geq 6,
\end{array}\right.
$$

then $\left(M^{n}, g\right)$ is isometric to a quotient of the round $\mathbb{S}^{n}$.

Corollary 1.4. Let $\left(M^{4}, g\right)$ be a 4-dimensional compact Riemannian manifold with harmonic Weyl curvature, positive scalar curvature and positive constant $\sigma_{2}\left(A_{g}\right)$. If

$$
|W|^{2}<8 \sigma_{2}\left(A_{g}\right),
$$

then $\left(M^{4}, g\right)$ is isometric to a quotient of the round $\mathbb{S}^{4}$ or a $\mathbb{C P}^{2}$ with the Fubini-Study metric.

Since for a 4-dimensional locally conformally flat Riemannian manifold $W \equiv 0$, we can directly get the following corollary from Corollary 1.4 
Corollary 1.5. Let $\left(M^{4}, g\right)$ be a 4-dimensional compact locally conformally flat Riemannian manifold with positive scalar curvature and positive constant $\sigma_{2}\left(A_{g}\right)$. Then $\left(M^{4}, g\right)$ is isometric to a quotient of the round $\mathbb{S}^{4}$.

Remark 1.6. Hu-Li-Simon [18] proved this similar result in which the Schouten tensor $A_{g}$ is semi-positive definite.

Corollary 1.7. Let $\left(M^{4}, g\right)$ be a 4-dimensional compact locally conformally flat Riemannian manifold with positive Yamabe constant and $\int_{M^{4}} \sigma_{2}\left(A_{g}\right)>0$. Then $\left(M^{4}, g\right)$ is conformal to a quotient of the round $\mathbb{S}^{4}$.

Remark 1.8. This result had been proved by Gursky [16], Xiao and the first author [13].

Corollary 1.9. Let $\left(M^{n}, g\right)(n \geq 5)$ be an n-dimensional compact locally conformally flat Riemannian manifold with positive scalar curvature and positive constant $\sigma_{2}\left(A_{g}\right)$. If

$$
\left|\operatorname{Ric}^{\circ}\right|^{2}<\frac{1}{n(n-1)} R^{2}
$$

then $\left(M^{n}, g\right)$ is isometric to a quotient of the round $\mathbb{S}^{n}$.

Remark 1.10. In [13], Xiao and the first author proved that for a compact locally conformally flat Riemannian manifold $\left(M^{n}, g\right)(n \geq 4)$ with positive constant scalar curvature, if $\left(\int_{M^{n}}|R i c|^{\frac{n}{2}}\right)^{\frac{2}{n}}<\frac{1}{n(n-1)} Y\left(M^{n},[g]\right)$, then $\left(M^{n}, g\right)$ is isometric to a quotient of the round $\mathbb{S}^{n}$. In [27], Pigola, Rigoli and Setti showed that for a complete locally conformally flat Riemannian manifold $\left(M^{n}, g\right)(n \geq 4)$ with positive constant scalar curvature, if $\mid$ Ric $\left.^{\circ}\right|^{2}<\frac{1}{n(n-1)} R^{2}$, $\left(M^{n}, g\right)$ is isometric to a quotient of the round $\mathbb{S}^{n}$.

Theorem 1.11. Let $\left(M^{n}, g\right)(n \geq 5)$ be an $n$-dimensional compact Riemannian manifold with harmonic Weyl curvature and positive Yamabe constant. If

$$
C(n)\left(\int_{M^{n}}|W|^{\frac{n}{2}}\right)^{\frac{2}{n}}+2 \sqrt{\frac{n-1}{n}}\left(\int_{M^{n}}|R i c|^{\frac{n}{2}}\right)^{\frac{2}{n}}<D(n) Y\left(M^{n},[g]\right),
$$

where

$$
D(n)= \begin{cases}\frac{3}{8}, & \text { for } n=5, \\ \frac{2}{n}, & \text { for } n \geq 6,\end{cases}
$$

then $\left(M^{n}, g\right)$ is locally conformally flat. Moreover, when $n=5$, the same result holds only assuming the weak inequality.

Remark 1.12. This result is a straightforward reworking of the first author's arguments on Theorem 1.10 in [11]. Although this result has been proved in [31], the pinching condition (1.6) is better for $n \geq 6$. When $n=4$, this result has been showed by the first author [10].

Acknowledgement: The authors are very grateful to Professor Haizhong Li for his guidance and constant support.

\section{Proofs of Lemmas}

In this section, in order to prove some main theorems in this article, we first give the following lemmas.

In what follows, we adopt, without further comment, the moving frame notation with respect to a chosen local orthonormal frame. And we adopt the Einstein convention. 
Let $M^{n}(n \geq 3)$ be an $n$-dimensional Riemannian manifold with harmonic Weyl curvature. Decomposing the Riemannian curvature tensor $R m$ into irreducible components yields

$$
\begin{aligned}
R_{i j k l}= & W_{i j k l}+\frac{1}{n-2}\left(R_{i k} \delta_{j l}-R_{i l} \delta_{j k}+R_{j l} \delta_{i k}-R_{j k} \delta_{i l}\right) \\
& -\frac{R}{(n-1)(n-2)}\left(\delta_{i k} \delta_{j l}-\delta_{i l} \delta_{j k}\right) \\
= & W_{i j k l}+\frac{1}{n-2}\left(\stackrel{\circ}{R}_{i k} \delta_{j l}-\stackrel{\circ}{R}_{i l} \delta_{j k}+\stackrel{\circ}{R}_{j l} \delta_{i k}-\stackrel{\circ}{R}_{j k} \delta_{i l}\right) \\
& +\frac{R}{n(n-1)}\left(\delta_{i k} \delta_{j l}-\delta_{i l} \delta_{j k}\right) \\
= & W_{i j k l}+\frac{1}{n-2}\left(A_{i k} \delta_{j l}-A_{i l} \delta_{j k}+A_{j l} \delta_{i k}-A_{j k} \delta_{i l}\right),
\end{aligned}
$$

where $R$ is the scalar curvature, $R_{i j k l}, W_{i j k l}, R_{i j}, \stackrel{\circ}{R}_{i j}$ and $A_{i j}$ denote the components of $R m$, the Weyl curvature tensor $W$, the Ricci tensor Ric, the trace-free Ricci tensor Ric $=R i c-\frac{R}{n} g$ and the Schouten tensor $A_{g}=$ Ric $-\frac{R}{2(n-1)} g$, respectively.

By the second Bianchi identity, we have

$$
\stackrel{\circ}{R}_{i j, k}-\stackrel{\circ}{R}_{i k, j}=R_{l i k j, l}+\frac{R_{, j}}{n} \delta_{i k}-\frac{R_{, k}}{n} \delta_{i j},
$$

and

$$
\stackrel{\circ}{R}_{i j, j}=\frac{n-2}{2 n} R_{, i} .
$$

Moreover, by the assumption that Weyl curvature is harmonic, we compute

$$
\begin{aligned}
0 & =W_{i j k l, l}=R_{i j k l, l}-\frac{1}{n-2}\left(\stackrel{\circ}{R}_{i k, l} \delta_{j l}-\stackrel{\circ}{R}_{i l, l} \delta_{j k}+\stackrel{\circ}{R}_{j l, l} \delta_{i k}-\stackrel{\circ}{R}_{j k, l} \delta_{i l}\right)-\frac{R_{, l}}{n(n-1)}\left(\delta_{i k} \delta_{j l}-\delta_{i l} \delta_{j k}\right) \\
& =\frac{n-3}{n-2}\left(\stackrel{\circ}{R}_{k i, j}-\stackrel{\circ}{R}_{k j, i}\right)-\frac{n-3}{2 n(n-1)}\left(R_{, i} \delta_{k j}-R_{, j} \delta_{k i}\right) .
\end{aligned}
$$

Thus we get

$$
\stackrel{\circ}{R}_{k i, j}-\stackrel{\circ}{R}_{k j, i}=\frac{n-2}{2 n(n-1)}\left(R_{, i} \delta_{k j}-R_{, j} \delta_{k i}\right) .
$$

Combining (2.3) with the second Bianchi identity, we obtain

$$
W_{i j k l, h}+W_{i j l h, k}+W_{i j h k, l}=0 .
$$

Lemma 2.1. Let $M^{n}(n \geq 4)$ be an $n$-dimensional complete Riemannian manifold with harmonic Weyl curvature. Then

$$
\frac{1}{2} \Delta|R i c|^{2}=|\nabla R i c|^{2}+\frac{n-2}{2(n-1)} \stackrel{\circ}{R}_{i j} R_{, i j}+W_{k i j l} \stackrel{\circ}{R}_{i j} \stackrel{\circ}{R}_{k l}+\frac{n}{n-2} \stackrel{\circ}{R}_{i j} \stackrel{\circ}{R}_{j l} \stackrel{\circ}{R}_{l i}+\frac{R}{n-1}|R i c|^{2}
$$

Proof. We compute

$$
\frac{1}{2} \Delta\left|R_{i c}^{\circ}\right|^{2}=|\nabla R i c|^{2}+\langle\text { Ric }, \Delta R i c\rangle=|\nabla R i c|^{2}+\stackrel{\circ}{R}_{i j} \stackrel{\circ}{R}_{i j, k k} .
$$


By Ricci identities and (2.3), we obtain

$$
\begin{aligned}
\stackrel{\circ}{R}_{i j, k k} & =\stackrel{\circ}{R}_{i k, j k}+\frac{n-2}{2 n(n-1)}\left(R_{, j k} \delta_{i k}-R_{, k k} \delta_{i j}\right) \\
& =\stackrel{\circ}{R}_{k i, k j}+\stackrel{\circ}{R}_{l i} R_{l k j k}+\stackrel{\circ}{R}_{k l} R_{l i j k}+\frac{n-2}{2 n(n-1)}\left(R_{, j i}-R_{, k k} \delta_{i j}\right) \\
& =\stackrel{\circ}{R}_{k k, i j}+\frac{n-2}{2 n(n-1)}\left(R_{, i j} \delta_{k k}-R_{, k j} \delta_{k i}\right)+\stackrel{\circ}{R}_{l i} R_{l j}+\stackrel{\circ}{R}_{k l} R_{l i j k}+\frac{n-2}{2 n(n-1)}\left(R_{, j i}-R_{, k k} \delta_{i j}\right) \\
& =\frac{n-2}{2 n(n-1)}\left(n R_{, i j}-R_{, k k} \delta_{i j}\right)+\stackrel{\circ}{R}_{l i} \stackrel{\circ}{R}_{l j}+\stackrel{\circ}{R}_{k l} R_{l i j k}+\frac{R}{n} \stackrel{\circ}{R}_{j i},
\end{aligned}
$$

which gives

$$
\begin{aligned}
& \frac{1}{2} \Delta|R i c|^{2}=|\nabla R i c|^{2}+\frac{n-2}{2(n-1)} \stackrel{\circ}{R}_{i j} R_{, i j}-\frac{n-2}{2 n(n-1)} R_{, k k} \delta_{i j} \stackrel{\circ}{R}_{i j}+\stackrel{\circ}{R}_{i j} \stackrel{\circ}{R}_{l i} \stackrel{\circ}{l}_{l j}+\stackrel{\circ}{R}_{i j} \stackrel{\circ}{R}_{k l} R_{l i j k}+\frac{R}{n}|R i c|^{2} \\
& =|\nabla R i c|^{2}+\frac{n-2}{2(n-1)} \stackrel{\circ}{R}_{i j} R_{, i j}+W_{k i j l} \stackrel{\circ}{R}_{i j} \stackrel{\circ}{R}_{k l}+\frac{n}{n-2} \stackrel{\circ}{R}_{i j} \stackrel{\circ}{R}_{j l} \stackrel{\circ}{R}_{l i}+\frac{R}{n-1}\left|R_{i c}\right|^{2} .
\end{aligned}
$$

This completes the proof of this Lemma.

Lemma 2.2. Let $M^{n}(n \geq 4)$ be an $n$-dimensional complete Riemannian manifold with harmonic Weyl curvature. Then

$$
\frac{1}{2} \Delta|W|^{2}=|\nabla W|^{2}+2 W_{i j k l}\left(2 W_{h j k m} W_{h i l m}-\frac{1}{2} W_{i j h m} W_{k l h m}\right)+\frac{2 R}{n}|W|^{2}+2 W_{i j k l} W_{i j k h} \stackrel{\circ}{R l}_{h l}
$$

Remark 2.3. (2.6) is a straightforward reworking of the first author's arguments on Lemma 2.4 in [11]. When $n=4$, it follows that $W_{i j k l} W_{i j k h}=\frac{1}{4}|W|^{2} \delta_{l h}$. Thus from (2.6) we have

$$
\frac{1}{2} \Delta|W|^{2}=|\nabla W|^{2}+2 W_{i j k l}\left(2 W_{h j k m} W_{h i l m}-\frac{1}{2} W_{i j h m} W_{k l h m}\right)+\frac{R}{2}|W|^{2} .
$$

Proof. By Ricci identities and (2.4), we obtain

$$
\begin{aligned}
\Delta|W|^{2}= & 2|\nabla W|^{2}+2\langle W, \Delta W\rangle=2|\nabla W|^{2}+2 W_{i j k l} W_{i j k l, m m} \\
= & 2|\nabla W|^{2}+2 W_{i j k l}\left(W_{i j k m, l m}+W_{i j m l, k m}\right) \\
= & 2|\nabla W|^{2}+4 W_{i j k l} W_{i j k m, l m} \\
= & 2|\nabla W|^{2}+4 W_{i j k l}\left(W_{i j k m, m l}+W_{h j k m} R_{h i l m}+W_{i h k m} R_{h j l m}+W_{i j h m} R_{h k l m}+W_{i j k h} R_{h m l m}\right) \\
= & 2|\nabla W|^{2}+4 W_{i j k l}\left(W_{h j k m} R_{h i l m}+W_{i h k m} R_{h j l m}+W_{i j h m} R_{h k l m}+W_{i j k h} R_{h m l m}\right) \\
= & 2|\nabla W|^{2}+4 W_{i j k l}\left(W_{h j k m} W_{h i l m}+W_{i h k m} W_{h j l m}+W_{i j h m} W_{h k l m}+W_{i j k h} W_{h m l m}\right) \\
& +\frac{4}{n-2} W_{i j k l}\left[W_{h j k m}\left(\stackrel{\circ}{R}_{h l} \delta_{i m}-\stackrel{\circ}{R}_{h m} \delta_{i l}+\stackrel{\circ}{R}_{i m} \delta_{h l}-\stackrel{\circ}{R}_{i l} \delta_{h m}\right)\right. \\
& +W_{i h k m}\left(\stackrel{\circ}{R}_{h l} \delta_{j m}-\stackrel{\circ}{R}_{h m} \delta_{j l}+\stackrel{\circ}{R}_{j m} \delta_{h l}-\stackrel{\circ}{R}_{j l} \delta_{h m}\right)+W_{i j h m}\left(\stackrel{\circ}{h}_{h l} \delta_{k m}-\stackrel{\circ}{R}_{h m} \delta_{k l}+\stackrel{\circ}{R}_{k m} \delta_{h l}-\stackrel{\circ}{R}_{k l} \delta_{h m}\right) \\
& \left.+W_{i j k h}\left(\stackrel{\circ}{R}_{h l} \delta_{m m}-\stackrel{\circ}{R}_{h m} \delta_{m l}+\stackrel{\circ}{R}_{m m} \delta_{h l}-\stackrel{\circ}{R}_{m l} \delta_{h m}\right)\right]+\frac{4 R}{n(n-1)} W_{i j k l}\left(W_{l j k i}+W_{i l k j}+W_{i j l k}\right)+\frac{4 R}{n}|W|^{2} \\
= & 2|\nabla W|^{2}+4 W_{i j k l}\left(2 W_{h j k m} W_{h i l m}-\frac{1}{2} W_{i j h m} W_{k l h m}\right)+\frac{4 R}{n}|W|^{2}+4 W_{i j k l} W_{i j k h} \stackrel{\circ}{R}_{h l .} .
\end{aligned}
$$

This completes the proof of this Lemma. 
Lemma 2.4. Let $M^{n}(n \geq 4)$ be an $n$-dimensional complete Riemannian manifold with harmonic Weyl curvature. Then

$$
\frac{1}{2} \Delta|W|^{2} \geq|\nabla W|^{2}-C(n)|W|^{3}-2 \sqrt{\frac{n-1}{n}}|W|^{2}\left|R^{\circ} i c\right|+\frac{2 R}{n}|W|^{2},
$$

where $C(n)$ is defined in Theorem 1.3.

Remark 2.5. When $n=4$, by Remark 2.3, we have

$$
\frac{1}{2} \Delta|W|^{2} \geq|\nabla W|^{2}-\frac{\sqrt{6}}{2}|W|^{3}+\frac{R}{2}|W|^{2} .
$$

Based on the above inequality, we obtain a integral rigidity for compact 4-manifold with harmonic Weyl curvature (see [10]).

Proof. By the algebraic inequality for $m$-trace-free symmetric two-tensors $T$, i.e., the eigenvalues $\lambda_{i}$ of $T$ satisfy $\left|\lambda_{i}\right| \leq \sqrt{\frac{m-1}{m}}|T|$ in [19], from (2.6) we get

$$
\frac{1}{2} \Delta|W|^{2} \geq|\nabla W|^{2}-2\left(2 W_{i j l k} W_{j h k m} W_{h i m l}+\frac{1}{2} W_{i j k l} W_{h m i j} W_{k l h m}\right)+\frac{2 R}{n}|W|^{2}-2 \sqrt{\frac{n-1}{n}}|W|^{2}\left|R^{\circ} i c\right| .
$$

Case 1. When $n=4$, it was proved in [19] that

$$
\left|2 W_{i j l k} W_{j h k m} W_{h i m l}+\frac{1}{2} W_{i j k l} W_{h m i j} W_{k l h m}\right| \leq \frac{\sqrt{6}}{4}|W|^{3} .
$$

Case 2. When $n=5$, Jack and Parker [21] have proved that $W_{i j k l} W_{h m i j} W_{k l h m}=2 W_{i j l k} W_{j h k m} W_{h i m l}$. By the algebraic inequality for $m$-trace-free symmetric two-tensors $T$, i.e., $\operatorname{tr}\left(T^{3}\right) \leq \frac{m-2}{\sqrt{m(m-1)}}|T|^{3}$ and equality holding if and only if $T$ can be diagonalized with $(n-1)$-eigenvalues equal to $\lambda$ in [19], we consider $W$ as a self-adjoint operator on $\wedge^{2} V$, and obtain

$$
\left|2 W_{i j l k} W_{j h k m} W_{h i m l}+\frac{1}{2} W_{i j k l} W_{h m i j} W_{k l h m}\right|=\frac{3}{2}\left|W_{i j k l} W_{h m i j} W_{k l h m}\right| \leq \frac{4}{\sqrt{10}}|W|^{3} .
$$

Case 3. When $n \geq 6$, considering $W$ as a self-adjoint operator on $S^{2} V$ and $\wedge^{2} V$, making use of the inequality proved by $\mathrm{Li}$ and Zhao [24] (see also [33]) and the Huisken's inequality in front, we have

$$
\begin{aligned}
\left|2 W_{i j l k} W_{j h k m} W_{h i m l}+\frac{1}{2} W_{i j k l} W_{h m i j} W_{k l h m}\right| & \leq 2\left|W_{i j l k} W_{j h k m} W_{h i m l}\right|+\frac{1}{2}\left|W_{i j k l} W_{h m i j} W_{k l h m}\right| \\
\leq & {\left[\frac{(n-2)}{\sqrt{n(n-1)}}+\frac{n^{2}-n-4}{2 \sqrt{(n-2)(n-1) n(n+1)}}\right]|W|^{3} . }
\end{aligned}
$$

From (2.8) and Cases 1, 2 and 3, we complete the proof of this Lemma.

Lemma 2.6. Let $M^{n}(n \geq 4)$ be an $n$-dimensional complete Riemannian manifold. The following estimate holds

$$
\left|-W_{i j k l} \stackrel{\circ}{R}_{i k} \stackrel{\circ}{R}_{j l}+\frac{n}{n-2} \stackrel{\circ}{R}_{i j} \stackrel{\circ}{R}_{j k} \stackrel{\circ}{R}_{k i}\right| \leq \sqrt{\frac{n-2}{2(n-1)}}\left(|W|^{2}+\frac{2 n}{n-2}|R i c|^{2}\right)^{\frac{1}{2}}\left|R_{i c}\right|^{2} .
$$

Remark 2.7. We follow these proofs of Proposition 2.1 in [7] and Lemma 4.7 in [3] to prove this lemma which was proved in [11]. For completeness, we also write it out. 
Proof. First, we have

$$
\begin{gathered}
(R i c \otimes g)_{i j k l}=\stackrel{\circ}{R}_{i k} g_{j l}-\stackrel{\circ}{R}_{i l} g_{j k}+\stackrel{\circ}{R}_{j l} g_{i k}-\stackrel{\circ}{R}_{j k} g_{i l}, \\
(\text { Ric } \otimes R i c)_{i j k l}=2\left(\stackrel{\circ}{R}_{i k} \stackrel{\circ}{R}_{j l}-\stackrel{\circ}{R}_{i l} \stackrel{\circ}{R}_{j k}\right),
\end{gathered}
$$

where $\otimes$ denotes the Kulkarni-Nomizu product. An easy computation shows

$$
\begin{gathered}
W_{i j k l} \stackrel{\circ}{R}_{i k} \stackrel{\circ}{R}_{j l}=\frac{1}{4} W_{i j k l}\left(R^{\circ} i c \otimes R i c\right)_{i j k l}, \\
\stackrel{\circ}{R}_{i j} \stackrel{\circ}{R}_{j k} \stackrel{\circ}{R}_{i k}=-\frac{1}{8}\left(R^{\circ} i c \otimes g\right)_{i j k l}\left(R^{\circ} i c \otimes R i c\right)_{i j k l} .
\end{gathered}
$$

Hence we get the following equation

$$
\text { (2.9) } \quad-W_{i j k l} \stackrel{\circ}{R}_{i k} \stackrel{\circ}{j}_{j l}+\frac{n}{(n-2)} \stackrel{\circ}{R}_{i j} \stackrel{\circ}{R}_{j k} \stackrel{\circ}{R}_{i k}=-\frac{1}{4}\left(W+\frac{n}{2(n-2)} R i c \otimes g\right)_{i j k l}(\operatorname{Ric} \otimes R i c)_{i j k l} \text {. }
$$

Since Ric $\otimes$ Ric has the same symmetries with the Riemannian curvature tensor, it can be orthogonally decomposed as

$$
\text { Ric } \otimes \text { Ric }=T+V^{\prime}+U^{\prime} .
$$

Here $T$ is totally trace-free, and

$$
\begin{gathered}
V_{i j k l}^{\prime}=-\frac{2}{n-2}\left(\operatorname{Ric}^{2} \otimes g\right)_{i j k l}+\frac{2}{n(n-2)} \mid \operatorname{Ric}^{2}(g \otimes g)_{i j k l}, \\
U_{i j k l}^{\prime}=-\frac{1}{n(n-1)} \mid \operatorname{Ric}^{\circ}(g \otimes g)_{i j k l},
\end{gathered}
$$

where $\left(\operatorname{Ric}^{2}\right)_{i k}=\stackrel{\circ}{R}_{i p} \stackrel{\circ}{R}_{k p}$. Taking the squared norm we obtain

$$
\begin{gathered}
\mid \text { Ric }^{\circ} \otimes \text { Ric }^{2}=8 \mid \text { Ric }^{4}-8 \mid \text { Ric }\left.^{2}\right|^{2}, \\
\left|V^{\prime}\right|^{2}=\frac{16}{n-2} \mid \text { Ric }\left.^{2}\right|^{2}-\frac{16}{n(n-2)} \mid \text { Ric }^{4}, \\
\left|U^{\prime}\right|^{2}=\frac{8}{n(n-1)} \mid \text { Ric }^{4} .
\end{gathered}
$$

In particular, one has

$$
|T|^{2}+\frac{n}{2}\left|V^{\prime}\right|^{2}=\left|R_{i c}^{\circ} \otimes R i c\right|^{2}+\frac{n-2}{2}\left|V^{\prime}\right|^{2}-\left|U^{\prime}\right|^{2}=\frac{8(n-2)}{n-1}\left|R^{\circ} i\right|^{4} .
$$

We now estimate the right hand side of (2.9). Using Cauchy-Schwarz inequality, 2.10) and the fact that $W$ and $T$ are totally trace-free we obtain

$$
\begin{aligned}
\mid\left.\left(W+\frac{n}{2(n-2)} R i c \otimes g\right)_{i j k l}(\text { Ric } \otimes R i c)_{i j k l}\right|^{2} & =\left|\left(W+\frac{n}{2(n-2)} R_{i c}^{\circ} \otimes g\right)_{i j k l}\left(T+V^{\prime}\right)_{i j k l}\right|^{2} \\
& =\left|\left(W+\frac{\sqrt{2 n}}{2(n-2)} R^{\circ} i c \otimes g\right)_{i j k l}\left(T+\sqrt{\frac{n}{2}} V^{\prime}\right)_{i j k l}\right|^{2} \\
& \leq\left|W+\frac{\sqrt{2 n}}{2(n-2)} R^{\circ} i c \otimes g\right|^{2}\left(|T|^{2}+\frac{n}{2}\left|V^{\prime}\right|^{2}\right) \\
& =\frac{8(n-2)}{n-1}\left|R_{i c}^{\circ}\right|^{4}\left(|W|^{2}+\frac{2 n}{n-2}\left|R_{i c}\right|^{2}\right) .
\end{aligned}
$$

This estimate together with (2.9) concludes this proof. 


\section{Proofs of Theorems} 2.

In this section, we prove our main theorems and corollaries by using lemmas in Section

Proposition 3.1. Let $\left(M^{n}, g\right)(n \geq 4)$ be an $n$-dimensional compact Riemannian manifold with harmonic Weyl curvature and positive scalar curvature. If

$$
\int_{M^{n}}|\nabla R i c|^{2} \geq \frac{(n-2)^{2}}{4 n(n-1)} \int_{M^{n}}|\nabla R|^{2}
$$

and

$$
|W|^{2}+\frac{2 n}{n-2}\left|R_{i c}^{\circ}\right|^{2}<\frac{2}{(n-2)(n-1)} R^{2}
$$

then $M^{n}$ is an Einstein manifold. In particular, if the pinching condition in (3.2) is weakened to

$$
|W|^{2}+\frac{2 n}{n-2}\left|R_{i c}\right|^{2}<\frac{4}{n^{2} C(n)^{2}} R^{2}
$$

then $M^{n}$ is isometric to a quotient of $\mathbb{S}^{n}$.

Proof. Integrating (2.5) by parts over $M^{n}$ and using (2.2) we get

$$
\begin{aligned}
0 & =\int_{M^{n}}|\nabla R i c|^{2}-\frac{n-2}{2(n-1)} \int_{M^{n}} \stackrel{\circ}{R}_{i j, j} R_{, i}+\int_{M^{n}} W_{k i j l} \stackrel{\circ}{R}_{i j} \stackrel{\circ}{R}_{k l}+\frac{n}{n-2} \int_{M^{n}} \stackrel{\circ}{R}_{i j} \stackrel{\circ}{R}_{j l} \stackrel{\circ}{R}_{l i}+\frac{1}{n-1} \int_{M^{n}} R|R i c|^{2} \\
& =\int_{M^{n}}|\nabla R i c|^{2}-\frac{(n-2)^{2}}{4 n(n-1)} \int_{M^{n}}|\nabla R|^{2}+\int_{M^{n}}\left(-W_{i k j l} \stackrel{\circ}{R}_{i j} \stackrel{\circ}{R}_{k l}+\frac{n}{n-2} \stackrel{\circ}{R}_{i j} \stackrel{\circ}{R}_{j l} \stackrel{\circ}{R}_{l i}\right)+\frac{1}{n-1} \int_{M^{n}} R|R i c|^{2} .
\end{aligned}
$$

Substituting (3.1) and Lemma 2.6 into (3.4) we get

$$
\begin{aligned}
0 & \geq-\sqrt{\frac{n-2}{2(n-1)}} \int_{M^{n}}\left(|W|^{2}+\frac{2 n}{n-2}\left|R_{i c}\right|^{2}\right)^{\frac{1}{2}} \mid \text { Ric }^{2}+\frac{1}{n-1} \int_{M^{n}} R \mid \text { Ric }^{2} \\
& =\int_{M^{n}}\left[\frac{R}{n-1}-\sqrt{\frac{n-2}{2(n-1)}}\left(|W|^{2}+\frac{2 n}{n-2}\left|R^{\circ} i c\right|^{2}\right)^{\frac{1}{2}}\right]\left|R_{i c}\right|^{2} .
\end{aligned}
$$

Hence the pinching condition (3.2) implies that Ric $=0$, i.e., $M^{n}$ is Einstein.

If (3.3) holds, then $M^{n}$ is Einstein. Thus it is easy to see that

$$
\frac{1}{2} \Delta|W|^{2} \geq|\nabla W|^{2}-C(n)|W|^{3}+\frac{2 R}{n}|W|^{2} .
$$

If $|W|<\frac{2}{n C(n)} R$, from (3.6) we have $|W|$ is a subharmonic function, and $|W|$ is constant. Hence from (3.6) we know that $W=0$, i.e., $M^{n}$ is locally conformally flat. By the rigidity result for positively curved Einstein manifolds (see Theorem 1.1 in [14]), we can directly get that $M^{n}$ is isometric to a quotient of the round $\mathbb{S}^{n}$.

Then we complete the proof of Propositon 3.1 . 
Proofs of Theorems 1.1 and 1.3. We compute

$$
\begin{aligned}
|\nabla A|^{2} & =|\nabla R i c|^{2}-\frac{1}{n-1}|\nabla R|^{2}+\frac{|\nabla R|^{2}}{4(n-1)^{2}} n \\
& =|\nabla R i c|^{2}-\frac{3 n-4}{4(n-1)^{2}}|\nabla R|^{2} \\
& =|\nabla R i c|^{2}+\frac{(n-2)^{2}}{4 n(n-1)^{2}}|\nabla R|^{2},
\end{aligned}
$$

and

$$
|\nabla t r A|^{2}=\frac{(n-2)^{2}}{4(n-1)^{2}}|\nabla R|^{2}
$$

Since $\sigma_{2}\left(A_{g}\right)$ is a positive constant, the inequality of Kato type due to Li and Simon[18, 25, 28], i.e.,

$$
|\nabla A|^{2} \geq|\nabla \operatorname{tr} A|^{2}
$$

holds. From (3.7) and (3.8), (3.9) implies that

$$
\int_{M^{n}}|\nabla R i c|^{2} \geq \frac{(n-2)^{2}}{4 n(n-1)} \int_{M^{n}}|\nabla R|^{2} .
$$

By (1.1), the pinching conditions (1.2) and (1.3) are equivalent to (3.2) and (3.3), respectively. By Proposition 3.1, we can complete the proofs of Theorems 1.1 and 1.3.

Remark 3.2. Since Riemannian manifolds with harmonic curvature has constant scalar curvature, (3.1) naturally holds. Proposition 3.1 improves Theorem 1.1 in [15].

Proof of Corollary 1.4. When $n=4$, the pinching condition (1.2) in Theorem 1.1 is reduced to (1.4) in Corollary 1.4. By Theorem 1.1, $M^{4}$ is Einstein. Thus by (1.1), (1.4) is equivalent to

$$
|W|^{2}<\frac{R^{2}}{3}
$$

By Theorem 1.8 in [10] (see also [11]), we obtain that $M^{4}$ is isometric to a quotient of the round $\mathbb{S}^{4}$ or a $\mathbb{C P}^{2}$ with the Fubini-Study metric. Then we complete the proof of Corollary 1.4

Proofs of Corollary 1.7. According to Theorem 1.1 in [8], there exists a conformal metric $\tilde{g}$ such that $\sigma_{2}\left(A_{\tilde{g}}\right)$ is a positive constant and $R_{\tilde{g}}$ is positive. Hence by Corollary 1.5 , we can complete the proof of Corollary 1.7.

Proof of Corollary 1.9. When $M^{n}$ is locally conformally flat, the pinching condition (1.2) in Theorem 1.1 is reduced to (1.5) in Corollary 1.9. By Theorem 1.1, $M^{n}$ is isometric to a quotient of the round $\mathbb{S}^{n}$. Then we complete the proof of Corollary 1.9

Proof of Theorem 1.11. By the Kato inequality $|\nabla W|^{2} \geq \frac{n+1}{n-1}|\nabla| W \|^{2}$ (see [4]) and (2.7), we have

$$
\frac{1}{2} \Delta|W|^{2} \geq\left.\frac{n+1}{n-1}|\nabla| W\right|^{2}-C(n)|W|^{3}-2 \sqrt{\frac{n-1}{n}}|W|^{2}|R i c|+\frac{2 R}{n}|W|^{2} .
$$

We rewrite (3.10) as

$$
|W| \Delta|W| \geq\left.\frac{2}{n-1}|\nabla| W\right|^{2}-C(n)|W|^{3}-2 \sqrt{\frac{n-1}{n}}|W|^{2}\left|R^{\circ} i c\right|+\frac{2 R}{n}|W|^{2}
$$


in the sense of distributions. Setting $u=|W|$, we compute from (3.11)

$$
\begin{aligned}
u^{\gamma} \Delta u^{\gamma} & =u^{\gamma}\left(\gamma(\gamma-1) u^{\gamma-2}|\nabla u|^{2}+\gamma u^{\gamma-1} \Delta u\right) \\
& =\frac{\gamma-1}{\gamma}\left|\nabla u^{\gamma}\right|^{2}+\gamma u^{2 \gamma-2} u \Delta u \\
& \geq\left(1-\frac{n-3}{(n-1) \gamma}\right)\left|\nabla u^{\gamma}\right|^{2}-C(n) \gamma u^{2 \gamma+1}-2 \sqrt{\frac{n-1}{n}} \gamma u^{2 \gamma}\left|R^{\circ} i c\right|+\frac{2 R}{n} \gamma u^{2 \gamma} .
\end{aligned}
$$

Integrating (3.12) by parts over $M^{n}$, it follows that

$0 \geq\left(2-\frac{n-3}{(n-1) \gamma}\right) \int_{M^{n}}\left|\nabla u^{\gamma}\right|^{2}-C(n) \gamma \int_{M^{n}} u^{2 \gamma+1}-2 \sqrt{\frac{n-1}{n}} \gamma \int_{M^{n}} u^{2 \gamma}|R i c|+\frac{2}{n} \gamma \int_{M^{n}} R u^{2 \gamma}$.

For $2-\frac{n-3}{(n-1) \gamma}>0$, by the definition of Yamabe constant and (3.13), we can obtain

$$
\begin{aligned}
& 0 \geq\left[\left(2-\frac{n-3}{(n-1) \gamma}\right) \frac{n-2}{4(n-1)} Y\left(M^{n},[g]\right)-C_{2}(n) \gamma\left(\int_{M^{n}}|W|^{\frac{n}{2}}\right)^{\frac{2}{n}}-2 \sqrt{\frac{n-1}{n}} \gamma\left(\int_{M^{n}}|R i c|^{\frac{n}{2}}\right)^{\frac{2}{n}}\right]\left(\int_{M^{n}}|W|^{\frac{2 n \gamma}{n-2}}\right)^{\frac{n-2}{n}} \\
&+\frac{8(n-1) \gamma+\frac{n(n-2)(n-3)}{(n-1) \gamma}-2 n(n-2)}{4 n(n-1)} \int_{M^{n}} R|W|^{2 \gamma} .
\end{aligned}
$$

Case 1. If $n=5$, choose $\gamma=\frac{1}{2}$. It follows from (3.14) that

$$
\begin{array}{r}
0 \geq\left[\frac{3}{8} Y\left(M^{n},[g]\right)-C_{2}(5)\left(\int_{M^{5}}|W|^{\frac{5}{2}}\right)^{\frac{2}{5}}-2 \sqrt{\frac{4}{5}}\left(\int_{M^{5}}|R i c|^{\frac{5}{2}}\right)^{\frac{2}{5}}\right]\left(\int_{M^{5}}|W|^{\frac{5}{3}}\right)^{\frac{3}{5}} \\
+\frac{1}{80} \int_{M^{5}} R|W| .
\end{array}
$$

From (3.15), the pinching condition (1.6) implies that $M^{5}$ is locally conformal flat. Moreover, we get the same conclusion if we assume just the weak inequality in (1.6).

Case 2. If $n \neq 5$, choose $\frac{1}{\gamma}=\frac{n-1}{n-3}\left(1+\sqrt{1-\frac{8(n-3)}{(n-2) n}}\right)$. From (3.14), we get

$$
0 \geq\left[\frac{2}{n} Y\left(M^{n},[g]\right)-C_{2}(n)\left(\int_{M^{n}}|W|^{\frac{n}{2}}\right)^{\frac{2}{n}}-2 \sqrt{\frac{n-1}{n}}\left(\int_{M^{n}}|R i c|^{\frac{n}{2}}\right)^{\frac{2}{n}}\right]\left(\int_{M^{n}}|W|^{\frac{2 n y}{n-2}}\right)^{\frac{n-2}{n}} .
$$

From (3.16), the pinching condition (1.6) implies that $M^{n}$ is locally conformal flat. Then we complete the proof of Theorem 1.11

\section{REFERENCES}

1. T. Aubin, Some Nonlinear Problems in Riemannian Geometry, Springer-Verlag, Berlin 1998.

2. A.L. Besse, Einstein manifolds, Springer-Verlag, Berlin, 1987.

3. V. Bour, Fourth order curvature flows and geometric applications. Arxiv: 1012.0342.

4. T. Branson, Kato constants in Riemannian geometry. Math. Res. Lett. 7 (2000), 245-261.

5. G. Carron, Some old and new results about rigidity of critical metric. Ann. Sc. Norm. Super. Pisa Cl. Sci. (5) 13 (2014), 1091-1113.

6. G. Catino, On conformally flat manifolds with constant positive scalar curvature. Proc. Amer. Math. Soc. 144 (2016), 2627-2634.

7. G. Catino, Integral pinched shrinking Ricci solitons. Adv. Math. 303 (2016), 279-294.

8. S.A. Chang, M.J. Gursky and P.C. Yang, A conformally invariant sphere theorem in four dimensions. Publ. Math. Inst. Hautes Études Sci. 98 (2003), 105-143. 
9. A. Derdziński, On compact Riemannian manifolds with harmonic curvature. Math. Ann. 259 (1982), 144152.

10. H.P. Fu, Four manifolds with postive Yamabe constant. Pacific J. Math. 296 (2018), 79-104.

11. H.P. Fu, On compact manifolds with harmonic curvature and positive scalar curvature. J. Geom. Anal. 27 (2017), 3120-3139.

12. H.P. Fu and J.K. Peng, Conformally flat Riemannian manifolds with finite $L^{p}$-norm curvature. Annali di Matematica 196 (2017), 1903-1912.

13. H.P. Fu and L.Q. Xiao, Some $L^{p}$ rigidity results for complete manifolds with harmonic curvature. Potential Analysis (2017) 48 (2018), 239-255.

14. H.P. Fu and L.Q. Xiao, Einstein manifolds with finite $L^{p}$-norm of the Weyl curvature. Differ. Geom. Appl. 53 (2017), 293-305.

15. H.P. Fu, G.B. Xu and Y.Q. Tao, Some remarks on Riemannian manifolds with parallel cotton tensor. Accepted by Colloquium Mathematicum.

16. M.J. Gursky, Locally conformally flat four-and six-manifolds of positive scalar curvature and positive Euler characteristic. Indiana Univ. Math. J. 43 (1994), 747-774.

17. E. Hebey and M. Vaugon, Effective $L^{p}$ pinching for the concircular curvature. J. Geom. Anal. 6 (1996), 531-553.

18. Z.J. Hu, H. Li and U. Simon, Schouten curvature functions on locally conformally flat Riemannian manifolds. J. Geom. 88 (2008), 75-100.

19. G. Huisken, Ricci deformation of the metric on a Riemannian manifold. J. Differential Geom. 21 (1985), $47-62$.

20. M. Itoh and H. Satoh, Isolation of the Weyl conformal tensor for Einstein manifolds. Proc. Jpn. Acad. A Math. Sci. 78 (2002), 140-142.

21. I. Jack and L. Parker, Linear independence of renormalisation counterterms in curved space-times of arbitrary dimensionality. J. Math. Phys. 28 (1987), 1137-1139.

22. S. Kim, Rigidity of noncompact complete manifolds with harmonic curvature. Manuscripta Math. 135 (2011), $107-116$.

23. M.J. Lee and T.H. Parker, The Yamabe problem. Bull. A. M. S. 17 (1987), 37-91.

24. A.M. Li and G.S. Zhao, Isolation phenomena for Riemannian manifolds whose Ricci curvature tensor are parallel. Acta Math. Sci. Ser. A Chin. Ed. 37 (1994), 19-24.

25. H. Li, Global rigidity theorems of hypersurfaces. Ark. Mat. 35 (1997) 327-351.

26. M. Okumura, Submanifolds and a pinching problem on the second fundamental tensors. Trans. Amer. Math. Soc. 178 (1973), 285-291.

27. S. Pigola, M. Rigoli and A.G. Setti, Some characterizations of space-forms. Trans. Amer. Math. Soc. 359 (2007), 1817-1828.

28. U. Simon, A further method in global differential geometry. Abh. Math. Semin. Univ. Hamburg 44 (1976) $52-69$.

29. M. Singer, Positive Einstein metrics with small $L^{n / 2}$-norm of the Weyl tensor. Differ. Geom. Appl. 2 (1992), 269-274.

30. S. Tachibana, A theorem on Riemannian manifolds of positive curvature operator. Proc. Japan Acad. 50 (1974), 301 Å-302.

31. H. Tran, On closed manifolds with harmonic Weyl curvature. Adv. Math. 322 (2017), 861-891.

32. H.W. Xu and E.T. Zhao, $L^{p}$ Ricci curvature pinching theorems for conformally flat Riemannian manifolds. Pacific J. Math. 245 (2010), 381-396.

33. Z.R. Zhou, Inequalities of Simons type and gaps for Yang-Mills fields. Ann. Glob. Anal. Geom. 48 (2015), 223-232.

Department of Mathematics, Nanchang University, Nanchang 330031, P. R. China

E-mail address: mathfu@126.com

Department of Mathematical Sciences, Tsinghua University, Beijing 100084, P. R. China

E-mail address: hhy15@mails.tsinghua.edu.cn 